\title{
Multiple source frequency-modulated continuous-wave optical reflectometry: theory and experiment
}

\author{
Arseny Vasilyev, ${ }^{1, *}$ Naresh Satyan, ${ }^{2}$ Shengbo $\mathrm{Xu},{ }^{2}$ \\ George Rakuljic, ${ }^{3}$ and Amnon Yariv ${ }^{1,2}$ \\ ${ }^{1}$ Department of Applied Physics, California Institute of Technology, 1200 East California \\ Boulevard 128-95, Pasadena, California 91125, USA \\ ${ }^{2}$ Department of Electrical Engineering, California Institute of Technology, 1200 East California \\ Boulevard 136-93, Pasadena, California 91125, USA \\ ${ }^{3}$ Telaris, Incorporated, 2118 Wilshire Boulevard \#238, Santa Monica, California 90403, USA \\ ${ }^{*}$ Corresponding author: vasilyev@ caltech.edu
}

Received 18 November 2009; revised 8 March 2010; accepted 11 March 2010; posted 12 March 2010 (Doc. ID 120148); published 26 March 2010

\begin{abstract}
We propose and demonstrate a novel approach to increase the effective bandwidth of a frequencymodulated continuous-wave (FMCW) ranging system. This is achieved by algorithmically stitching together the swept spectra of separate laser sources. The result is an improvement in the range resolution proportional to the increase in the swept-frequency range. An analysis of this system as well as the outline of the stitching algorithm are presented. Using three distinct swept-frequency optical waveforms, we experimentally demonstrate a threefold improvement in the range resolution of a three-sweep approach over the conventional FMCW method. (c) 2010 Optical Society of America
\end{abstract}

OCIS codes: $110.4500,140.5960,280.3640$.

\section{Introduction}

The technique of optical frequency-modulated continuous-wave (FMCW) reflectometry, also known as swept-source optical coherence tomography (SS-OCT) and optical frequency domain imaging in the biomedical optics community, has found applications in lidar [1], biomedical imaging [2,3], noncontact profilometry [4], and biometrics [5], due to its high dynamic range and data acquisition that does not require high-speed electronics [6]. The key component of an FMCW experiment is the sweptfrequency (chirped) laser, since its performance directly affects important system metrics. Specifically, the range resolution and the ranging depth are inversely proportional to the laser frequency tuning range

0003-6935/10/101932-06\$15.00/0

(C) 2010 Optical Society of America and linewidth, respectively [6]. Mechanically tunable extended cavity lasers with large frequency excursions of about $10 \mathrm{THz}$ have been used in medical tomographic applications to achieve range resolutions of about $10 \mu \mathrm{m}[2,7]$. However, linewidths of tens of $\mathrm{GHz}$, which are typical for such devices, limit ranging depths to just a few $\mathrm{mm}$. Moreover, the mechanical nature of the frequency tuning limits the scan repetition rate to a few hundred $\mathrm{kHz}$. Commercially available semiconductor laser (SCL) diodes, on the other hand, offer superior sub-MHz linewidths, corresponding to ranging depths of a few hundred $m$, and can be frequency tuned with current at rates of a few $\mathrm{GHz}$. The small size of such devices makes them attractive for handheld applications, while batch processing dramatically cuts production costs. The drawback of SCL diodes is the comparatively small tuning range of several hundred $\mathrm{GHz}$ at best. In this paper we present a new approach, multiple 
source FMCW (MS-FMCW) reflectometry, which combines multiple lasers in a manner that augments the system total optical bandwidth to the sum of the individual (laser) bandwidths and, thus, leads to a corresponding decrease in the smallest resolvable feature separation, while keeping the ranging depth and scan speed unchanged. The key to this technique is sweeping the sources over distinct, but adjacent, regions of the optical spectrum, so as to approximate a single sweep of greater bandwidth. A related method for range resolution improvement has recently been reported [8], where the chirped sidebands of the discrete frequencies radiated by a mode-locked laser are combined using feedback to create a phasecoherent continuous-frequency wideband chirp. In contrast, this paper focuses on an analytical method that can tolerate the presence of discontinuities in the frequency sweep, enabling a significantly simpler (and cheaper) combination of multiple sources for resolution improvement.

\section{FMCW Analysis}

Consider the FMCW experiment shown in Fig. 1. If the target consists of a single perfect reflector, the system is equivalent to a Mach-Zehnder interferometer (MZI). The goal of the measurement is to determine the time delay $\tau$ between the two arms, which is linearly related to the target range. This is accomplished by exciting the system with a sweptfrequency source. For simplicity, we consider a laser whose frequency changes linearly with time. In the experimental section of this paper, this linearity is imposed by a feedback system [9]. Additionally, we assume that $\tau$ is much smaller than the laser coherence time, so that any phase noise contribution may be neglected. The normalized electric field of the source is then given by

$$
e(t)=\operatorname{rect}\left(\frac{t-T / 2}{T}\right) \cos \left(\phi_{0}+\omega_{0} t+\frac{\xi t^{2}}{2}\right),
$$

where $T$ is the scan duration, $\xi$ is the slope of the optical chirp, and $\phi_{0}$ and $\omega_{0}$ are the initial phase and frequency, respectively. The rect function is defined by

$$
\operatorname{rect}(x) \equiv \begin{cases}0, & |x|>1 / 2 \\ 1 / 2, & |x|=1 / 2 \\ 1, & |x|<1 / 2\end{cases}
$$

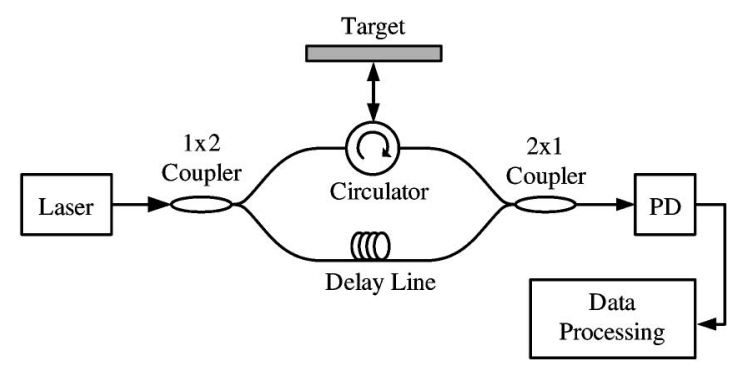

Fig. 1. Schematic of an FMCW ranging experiment: PD, photodetector.
The instantaneous optical frequency is given by the time derivative of the argument of the cosine in Eq. (1):

$$
\omega(t)=\omega_{0}+\xi t
$$

The total frequency excursion of the source (in $\mathrm{Hz}$ ) is then given by $B=\xi T / 2 \pi$. The normalized photocurrent is equal to the time averaged intensity of the incident beam and is given by

$$
\begin{aligned}
i(t) & =\left\langle|e(t)+\operatorname{Re}(t-\tau)|^{2}\right\rangle \\
& =\operatorname{Rrect}\left(\frac{t-T / 2}{T}\right) \cos \left[(\xi \tau) t+\omega_{0} \tau-\frac{\xi \tau^{2}}{2}\right],
\end{aligned}
$$

where $R$ is the target reflectivity, and no dc terms were included. The averaging is done over a time interval that is much longer than an optical cycle, yet much shorter than the period of the cosine in Eq. (4). As will become apparent later, it is convenient to work in the optical frequency domain, so we use Eq. (3) to rewrite the photocurrent as a function of $\omega$ :

$$
\begin{aligned}
y(\omega) & \equiv i\left(\frac{\omega-\omega_{0}}{\xi}\right) \\
& =R \operatorname{rect}\left(\frac{\omega-\omega_{0}-\pi B}{2 \pi B}\right) \cos \left(\omega \tau-\frac{\xi \tau^{2}}{2}\right) .
\end{aligned}
$$

The delay $\tau$ is found by taking the Fourier transform (FT) of $y(\omega)$ with respect to the variable $\omega$, which yields a normalized sinc function, $\operatorname{sinc}(x) \equiv$ $\sin (\pi x) / \pi x$, centered at the delay $\tau$ :

$$
\begin{aligned}
Y(\zeta)= & \pi B R \exp \left(-j \frac{\xi \tau^{2}}{2}\right) \exp \left[-j(\zeta-\tau)\left(\omega_{0}\right.\right. \\
& +\pi B)] \operatorname{sinc}[B(\zeta-\tau)]
\end{aligned}
$$

where $\zeta$ is the independent variable of the FT of $y(\omega)$, and has units of time. In this paper we use capital letters to denote the FT of the corresponding lowercase function. Additionally, we only consider positive Fourier frequencies since the signals of interest are purely real.

The range resolution, a measure of the smallest resolvable feature separation in the axial direction, is traditionally chosen to correspond to the coordinate of the first null of the sinc function in Eq. (6) [10]. This happens at $\zeta=\tau+1 / B$, which corresponds to a free space range resolution

$$
\Delta d=\frac{c}{2 B}
$$

where $c$ is the speed of light. An equivalent metric of the resolution of the FMCW system is the full width at half-maximum (FWHM) of the sinc function, given by 


$$
\mathrm{FWHM} \approx \frac{3.79}{\pi B}=\Delta d \frac{7.58}{\pi c} .
$$

Let us now consider the following view of an FMCW imaging system. The target is characterized by some underlying function of the optical frequency, $y_{\text {target }}(\omega)$, given by

$$
y_{\text {target }}(\omega)=\sum_{n} R_{n} \cos \left(\omega \tau_{n}-\frac{\xi \tau_{n}^{2}}{2}\right),
$$

where $\tau_{n}$ and $R_{n}$ are the delays and reflectivities of the multiple reflectors that comprise the target. In deriving Eq. (9), we have assumed highly transparent reflectors $\left(R_{n} \ll 1\right)$ and ignored interference between reflected beams. The range measurement is then given by

$$
y(\omega)=a(\omega) y_{\text {target }}(\omega),
$$

where $a(\omega)$ is the rectangular window function, as in Eq. (5). The function $y_{\text {target }}(\omega)$ contains all the information about the target, and perfect resolution is obtained if $y_{\text {target }}(\omega)$ is known for all values of the optical frequency $\omega$. The measurement in Eq. (10) gives us partial information about $y_{\text {target }}(\omega)$, collected over the frequency excursion defined by $a(\omega)$, resulting in a nonzero $\Delta d$.

We next develop the theory of MS-FMCW reflectometry, in which multiple sources sweep over distinct regions of the optical spectrum. The motivation for this approach is that the use of multiple sources allows us to further characterize $y_{\text {target }}(\omega)$, resulting in an increase in the effective $B$ and a corresponding decrease in $\Delta d$.

\section{Multiple Source Analysis}

Taking the FT of Eq. (10) and using Eq. (9), we arrive at the expression

$$
Y(\zeta)=\frac{1}{2} \sum_{n} R_{n} \exp \left(-j \frac{\xi \tau_{n}^{2}}{2}\right) A\left(\zeta-\tau_{n}\right)
$$

which has peaks at $\zeta=\tau_{n}$. The shape of the peaks is determined by the FT $A(\zeta)$ of the window function. We model the use of $N$ multiple sources with a window function $a_{N}(\omega)$ that is comprised of $N$ nonoverlapping rectangular sections, as shown in the top panel of Fig. 2(a). The approach is easily modified to include overlapping sections. The $k$ th sweep originates at $\omega_{0, k}$ and is characterized by an angular frequency excursion $2 \pi B_{k}$, where $k=1, \ldots, N$. As illustrated in the middle and bottom panels of Fig. 2(a), $a_{N}(\omega)$ can be decomposed into a rectangular window with width $2 \pi \tilde{B} \equiv 2 \pi\left[\sum_{k=1}^{N} B_{k}+\sum_{k=1}^{N-1} \delta_{k}\right]$ and a set of thin rectangular sections (gaps). Each gap represents the frequency range $2 \pi \delta_{k}$ between the end of the $k$ th sweep and the beginning of the $(k+1)$ th sweep, across which no photocurrent is measured. Amplitudes of the $\zeta$-domain FTs of the functions in Fig. 2(a) are shown in Fig. 2(b). We observe that if the gaps chosen are sufficiently small, their effect in the $\zeta$ domain can
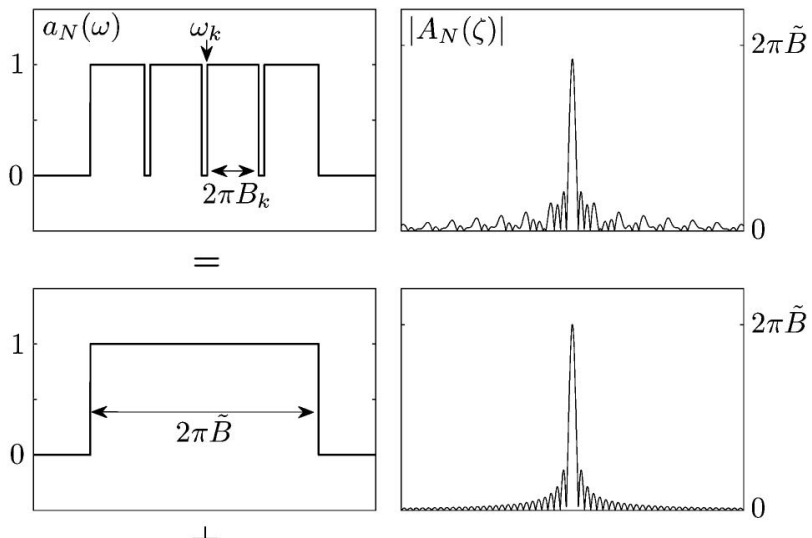

(a)

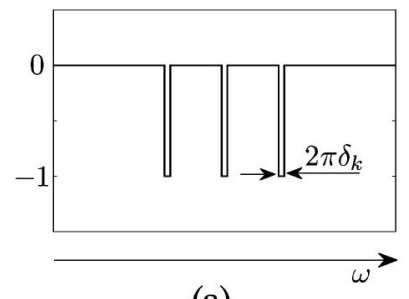

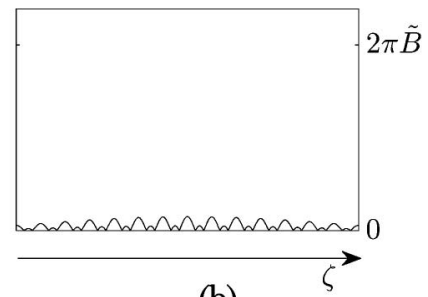

(b)
Fig. 2. Multiple source model: (a) $\omega$-domain description. The top panel shows a multiple source window function $a_{N}(\omega)$. This function may be decomposed into the sum of a single source window function (middle panel) and a function that describes the intersweep gaps (bottom panel). (b) $\zeta$-domain description. The three figures show the amplitudes of the $\zeta$-domain FTs of the corresponding functions from (a).

be treated as a small perturbation of the single sweep of bandwidth $\tilde{B}$. Therefore, an $N$-source sweep is described by

$$
\begin{aligned}
a_{N}(\omega)= & \operatorname{rect}\left(\frac{\omega-\omega_{0,1}-\pi \tilde{B}}{2 \pi \tilde{B}}\right) \\
& -\sum_{k=1}^{N-1} \operatorname{rect}\left(\frac{\omega-\omega_{0, k+1}+\pi \delta_{k}}{2 \pi \delta_{k}}\right)
\end{aligned}
$$

in the $\omega$ domain, and by

$$
\begin{aligned}
A_{N}(\zeta)= & 2 \pi \tilde{B} \exp \left[-j \zeta\left(\omega_{0,1}+\pi \tilde{B}\right)\right] \operatorname{sinc}(\zeta \tilde{B}) \\
& -2 \pi \sum_{k=1}^{N-1} \delta_{k} \exp \left[-j \zeta\left(\omega_{0, k+1}-\pi \delta_{k}\right)\right] \operatorname{sinc}\left(\zeta \delta_{k}\right)
\end{aligned}
$$

in the $\zeta$ domain. To find the range resolution, we find the first null of Eq. (13). Expanding near $\zeta=1 / \tilde{B}$ and using the approximation $\sum_{k=1}^{N-1} \delta_{k} \ll \sum_{k=1}^{N} B_{k}$ yields

$$
\begin{aligned}
\zeta_{\text {null }}^{-1}= & \mid \tilde{B} \exp \left[-j \zeta_{\text {null }}\left(\omega_{0,1}+\pi \tilde{B}\right)\right] \\
& +\sum_{k=1}^{N-1} \delta_{k} \exp \left[-j \zeta_{\text {null }}\left(\omega_{0, k+1}-\pi \delta_{k}\right)\right] \mid
\end{aligned}
$$


Equation (14) can be solved numerically to find $\zeta_{\text {null }}$. We note that an upper bound on $\zeta_{\text {null }}$, and consequently on the range resolution, may be obtained by applying the triangle inequality to Eq. (14), to yield

$$
\Delta d_{\mathrm{MS}-\mathrm{FMCW}} \leq \frac{c}{2 \sum_{k=1}^{N} B_{k}} .
$$

The conclusion is that by sweeping over distinct regions of the optical spectrum, we collect enough information about the target to arrive at a range resolution equivalent to the total traversed optical bandwidth, provided that the said bandwidth is much greater than the intersweep gaps.

\section{Stitching}

We next consider the problem of stitching, that is, synthesizing a measurement with enhanced resolution using photocurrents collected from multiple sweeps. In the preceding sections, we have mapped photocurrents from the time domain to the optical frequency domain. Since the optical frequency is linear in time, this mapping involves first scaling the time axis by the chirp slope, and then translating the data to the correct initial frequency. Whereas the rate of each chirp is precisely controlled [9], the starting sweep frequencies are not known with sufficient accuracy. To reflect this uncertainty, we omit the translation step, so that the data collected during the $k$ th scan is given by

$$
y_{k}(\omega)=\operatorname{rect}\left(\frac{\omega-\pi B_{k}}{2 \pi B_{k}}\right) y_{\text {target }}\left(\omega+\omega_{0, k}\right) .
$$

The stitched measurement, given by $y_{\text {stitched }}=$ $a_{N}(\omega) y_{\text {target }}(\omega)$, can be written in terms of functions $y_{k}(\omega)$ using Eq. (12):

$$
y_{\text {stitched }}(\omega)=\sum_{k=1}^{N} y_{k}\left(\omega-\omega_{0, k}\right) .
$$

The magnitude of the FT of Eq. (17) may be used for target recognition and is given by

$$
\left|Y_{\text {stitched }}(\zeta)\right|=\left|\sum_{k=1}^{N} \exp \left[-j 2 \pi \zeta \sum_{l=1}^{k-1}\left(B_{l}+\delta_{l}\right)\right] Y_{k}(\zeta)\right|
$$

The uncertainty in the starting frequencies manifests itself as an uncertainty in the intersweep gaps. To recover the gaps, we use a known reference target along with the target of interest, as shown in Fig. 3 . By analyzing the data collected from the reference target, we are able to extract the parameters $\delta_{k}$, and stitch together the target of interest measurement, according to Eq. (18).

To develop a gap recovery algorithm, we examine a two-sweep system with a single gap $\delta$. The case of more than two sources may then be treated by apply-

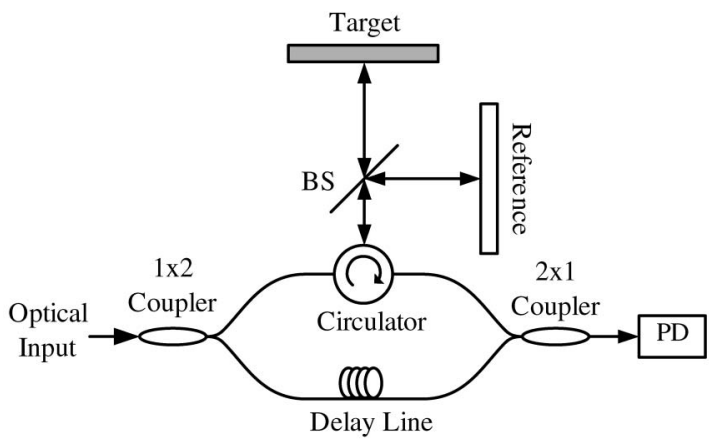

Fig. 3. Schematic of an MS-FMCW ranging experiment. A reference target is imaged along with the target of interest, so that the intersweep gaps may be recovered: BS, beam splitter; PD, photodetector.

ing this algorithm to adjacent sweeps in a pairwise manner. For simplicity we consider sweeps of equal slopes $\xi$ and durations $T$ and, therefore, bandwidths $B$. Suppose the known reference target consists of a single reflector with reflectivity $R_{a}$, located at the delay $\tau_{a}$. The experiment of Fig. 3 generates two photocurrents, of the form of Eq. (4). The initial photocurrent phase depends on the starting frequency of the corresponding sweep and will change as the intersweep gap varies. Therefore, by considering the phase difference between the two photocurrents, we can calculate the value of the gap. Formally, let us evaluate the FT of the $k$ th photocurrent, at the maximum of the reference target peak. Using Eqs. (9) and (16),

$$
Y_{k}\left(\tau_{a}\right)=\pi B_{k} R_{a} \exp \left[-j \xi \frac{\tau_{a}^{2}}{2}+j \omega_{0, k} \tau_{a}\right], \quad k=1,2 .
$$

The ratio of the two expressions in Eq. (19) yields the phase difference between the photocurrents:

$$
\psi_{a} \equiv \frac{Y_{1}\left(\tau_{a}\right)}{Y_{2}\left(\tau_{a}\right)}=\exp \left[-j 2 \pi \tau_{a}(B+\delta)\right]
$$

Given the reference reflector delay $\tau_{a}$ and the frequency excursion $B$, the gap may be found using

$$
\arg \left[\exp \left(j 2 \pi \tau_{a} B\right) \psi_{a}\right]=-2 \pi \tau_{a} \delta .
$$

The phase of a complex number can only be extracted modulo $2 \pi$, so that Eq. (21) can only be used to recover $\delta$ with an ambiguity of $1 / \tau_{a}$. Therefore, the gap needs to be known to within $1 / \tau_{a}$ before Eq. (21) may be applied. For example, if the nominal gap is only known to an accuracy of $10 \mathrm{GHz}$, we need $1 / \tau_{a}>10 \mathrm{GHz}$. However, the nonzero linewidth of the source generates errors in the phase measurement $\psi_{a}$ in Eq. (20) [11]. According to Eq. (21), the corresponding error in the gap calculation is inversely proportional to $\tau_{a}$, and a large $\tau_{a}$ is necessary to calculate $\delta$ accurately. 
To overcome this issue, we use two reflectors $\tau_{a}$ and $\tau_{b}$ and express the gap size as a function of the reflector separation. We define two phase factors

$$
\psi_{n} \equiv \frac{Y_{1}\left(\tau_{n}\right)}{Y_{2}\left(\tau_{n}\right)}, \quad n=a, b
$$

and calculate the two-reflector analog of Eq. (21):

$$
\arg \left\{\exp \left[j 2 \pi\left(\tau_{a}-\tau_{b}\right) B\right] \frac{\psi_{a}}{\psi_{b}}\right\}=-2 \pi\left(\tau_{a}-\tau_{b}\right) \delta
$$

From Eq. (23), $1 /\left|\tau_{a}-\tau_{b}\right|$ can be chosen to be $>10 \mathrm{GHz}$ to determine the value of $\delta$. The error in this calculation is proportional to $1 /\left|\tau_{a}-\tau_{b}\right|$. The accuracy of the gap calculation can now be improved by using Eq. (21), which yields a new value of $\delta$ with a lower error proportional to $1 / \tau_{a}$. Depending on system noise levels, more stages of evaluation of $\delta$ using more than two reference reflectors may be utilized to achieve better accuracy in the calculations.

A potential system architecture employing the stitching technique for high-resolution MS-FMCW is shown in Fig. 4. The optical sources are multiplexed and used to image a target and a reference, as discussed above. The optical output is demultiplexed and measured using a set of photodetectors to generate the photocurrents of Eq. (16). The reference data are processed and used to stitch a target measurement of improved resolution. The multiplexing may be performed in time or optical frequency, or a combination of the two. The real power of the MS-FMCW technique then lies in its scalability. One envisions a system that combines cheap off-theshelf SCLs to generate a swept-frequency ranging measurement that features an excellent combination of range resolution, scan speed, and imaging depth.

\section{Experimental Results}

We demonstrated the MS-FMCW technique using a highly linear optoelectronic swept-frequency source that chirps $100 \mathrm{GHz}$ around a central wavelength of $1550 \mathrm{~nm}$ in a $1 \mathrm{~ms}$ long scan [9]. The optoelectronic source is comprised of an SCL coupled to an MZI, a photodetector at the MZI output, and electronic filters that process the photocurrent and feed it back into the SCL. The MZI measures the instantaneous chirp slope, and the feedback loop ensures a perfectly linear chirp. We note that a specialized source is not necessary, and chirp nonlinearity may be compensated for by sampling the photocurrent uniformly in optical frequency [3].

We used the configuration of Fig. 3 with a $1.0 \mathrm{~mm}$ microscope slide target and a two reflector reference characterized by $1 /\left|\tau_{a}-\tau_{b}\right| \sim 10 \mathrm{GHz} \quad(\sim 3 \mathrm{~cm}$ free space separation). This reference was chosen to accommodate the accuracy with which the gaps are initially known $(\sim 1 \mathrm{GHz})$. We tuned the SCL temperature through three set points to generate three $100 \mathrm{GHz}$ sweeps with different starting frequencies. These sweeps were sequentially launched into the experiment, and the corresponding photocurrents were recorded. Using the two-step procedure described in Section 4, the gaps between the sweeps were calculated to be $1.89 \mathrm{GHz}$ and $0.72 \mathrm{GHz}$.

These gap values were used in Eq. (18) to stitch the three measured photocurrents, and the results are plotted in Fig. 5. Figure 5(a) shows the single sweep and stitched multiple sweep spectra for one of the reference reflectors. The FWHMs are $12.17 \mathrm{ps}$ and $4.05 \mathrm{ps}$ for the single and multiple source cases, respectively. Using Eq. ( $\underline{8})$ we calculate the free space range resolutions to be $1.51 \mathrm{~mm}$ and $500 \mu \mathrm{m}$, respectively. The threefold range resolution enhancement is consistent with Eq. (15). Figure 5(b) shows the target measurements. The two peaks in the single-scan spectrum, corresponding to reflections from the two microscope slide facets, are barely resolved. This is consistent with the theoretical range resolution in glass of $1 \mathrm{~mm}$ for a $100 \mathrm{GHz}$ sweep. The stitched curve shows two prominent peaks, demonstrating our improved ability to resolve two closely spaced targets. The measured peak separation of $10 \mathrm{ps}$ is the roundtrip delay between the two slide facets and, indeed, corresponds to a glass thickness of $1 \mathrm{~mm}$.

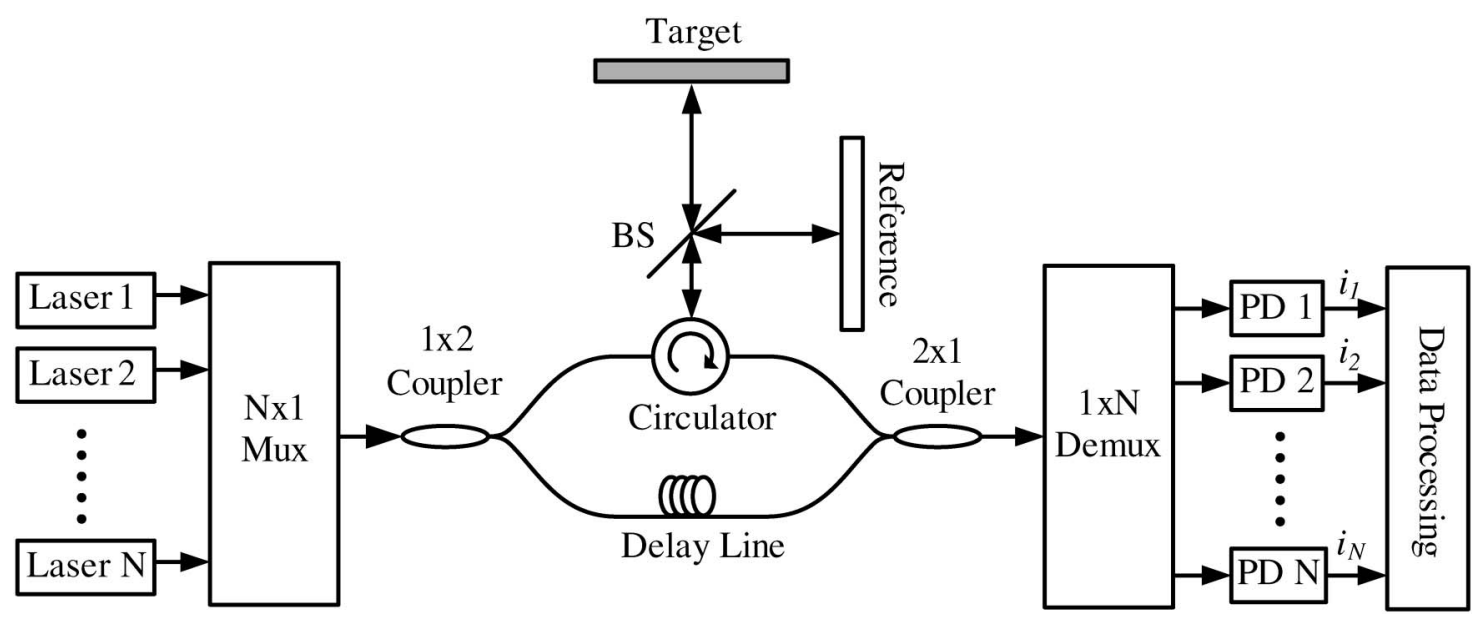

Fig. 4. Proposed MS-FMCW system architecture: BS, beam splitter; PD, photodetector. 

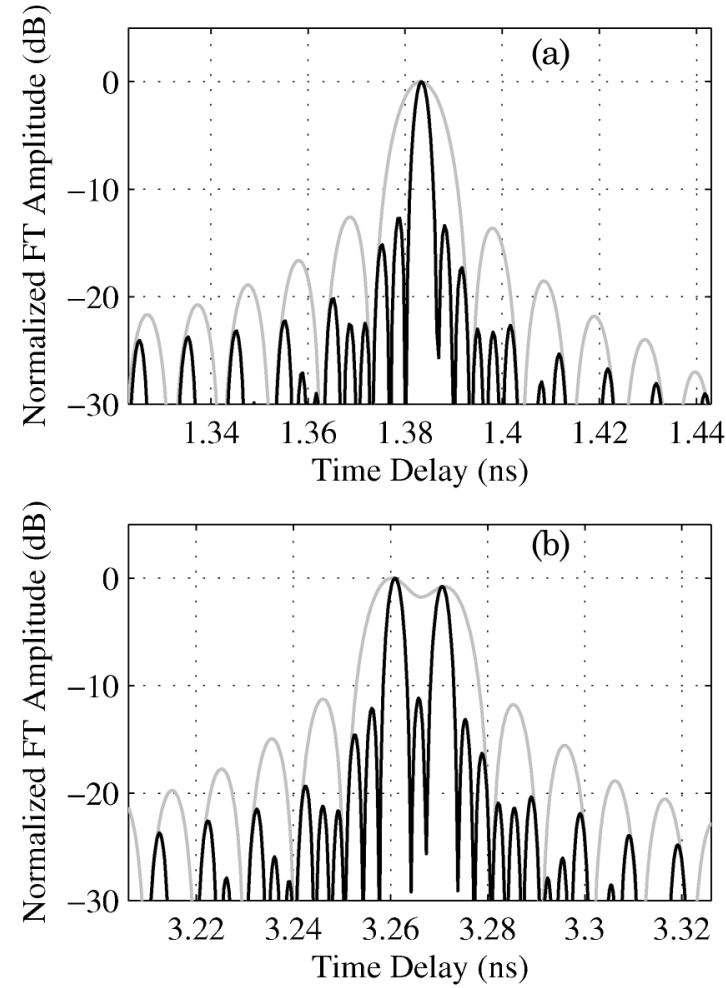

Fig. 5. Experimental results. The gray and black curves correspond to single-sweep and stitched three-sweep measurements, respectively: (a) single reflector spectrum and (b) glass slide spectrum. The peaks correspond to reflections from the two air/glass interfaces. The slide thickness is $1 \mathrm{~mm}$.

\section{Summary}

We have analyzed and demonstrated a novel variant of the FMCW optical imaging technique. This method combines multiple lasers that sweep over distinct but adjacent regions of the optical spectrum, in order to record a measurement with increased effective optical bandwidth and a corresponding improvement in the range resolution. The MS-FMCW technique is highly scalable and is a promising approach to realize a wide-bandwidth swept-frequency imaging system that inherits the speed and coherence of the SCL. While we have demonstrated the stitching of three $100 \mathrm{GHz}$ sweeps using DFB SCLs in our proof-of-concept experiment, MS-FMCW reflectometry is not tied to any particular laser type and may be used to combine wideband swept sources, such as vertical cavity surface emitting lasers or conventional sources for SS-OCT, to push range resolutions beyond the state of the art.

This work was supported by the Defense Advanced Research Projects Agency (DARPA) Microsystems Technology Office (R. Esman) and the California Institute of Technology Lee Center for Advanced Networking.

\section{References}

1. A. Dieckmann, "FMCW-lidar with tunable twin-guide laser diode," Electron. Lett. 30, 308-309 (1994).

2. S. H. Yun, G. J. Tearney, B. J. Vakoc, M. Shishkov, W. Y. Oh, A. E. Desjardins, M. J. Suter, R. C. Chan, J. A. Evans, I.-K. Jang, N. S. Nishioka, J. F. de Boer, and B. E. Bouma, "Comprehensive volumetric optical microscopy in vivo," Nat. Med. 12, 1429-1433 (2007).

3. M. A. Choma, K. Hsu, and J. A. Izatt, "Swept source optical coherence tomography using an all-fiber $1300 \mathrm{~nm}$ ring laser source," J. Biomed. Opt. 10, 044009 (2005).

4. C. Ndiaye, T. Hara, and H. Ito, "Profilometry using a frequency-shifted feedback laser," in Proceedings of the Conference on Lasers and Electro-Optics, 2005 (IEEE, 2005), Vol. 3, pp. 1757-1759.

5. S. K. Dubey, T. Anna, C. Shakher, and D. S. Mehta, "Fingerprint detection using full-field swept-source optical coherence tomography," Appl. Phys. Lett. 91, 181106 (2007).

6. M.-C. Amann, T. Bosch, M. Lescure, R. Myllylä, and M. Rioux, "Laser ranging: a critical review of usual techniques for distance measurement," Opt. Eng. 40, 10-19 (2001).

7. S. H. Yun, C. Boudoux, G. J. Tearney, and B. E. Bouma, "Highspeed wavelength-swept semiconductor laser with a polygonscanner-based wavelength filter," Opt. Lett. 28, 1981-1983 (2003).

8. K. W. Holman, D. G. Kocher, and S. Kaushik, MIT/LL development of broadband linear frequency chirp for high resolution ladar Proc. SPIE 6572, 65720J (2007).

9. N. Satyan, A. Vasilyev, G. Rakuljic, V. Leyva, and A. Yariv, "Precise control of broadband frequency chirps using optoelectronic feedback," Opt. Express 17, 15991-15999 (2009).

10. I. Komarov and S. Smolskiy, Fundamentals of Short-Range FM Radar (Artech, 2003).

11. E. Strzelecki, D. Cohen, and L. Coldren, "Investigation of tunable single frequency diode lasers for sensor applications," J. Lightwave Technol. 6, 1610-1618 (1988). 